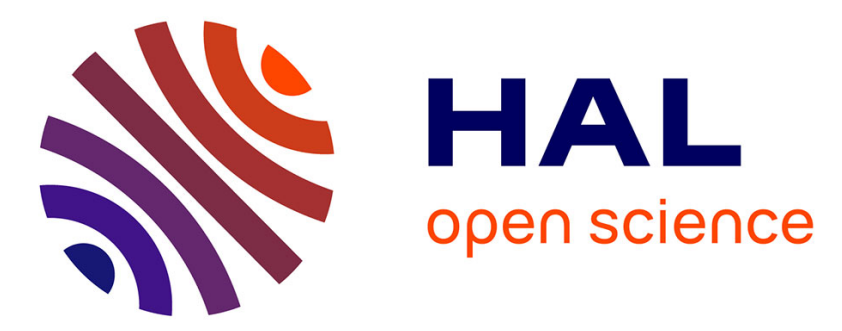

\title{
Competitive diffusion of gases in a zeolite using proton NMR and a slice selection procedure
}

\author{
S. Leclerc, M. Petryk, D. Canet, J. Fraissard
}

\section{To cite this version:}

S. Leclerc, M. Petryk, D. Canet, J. Fraissard. Competitive diffusion of gases in a zeolite using proton NMR and a slice selection procedure. Catalysis Today, 2012, 187 (1), pp.104 - 107. 10.1016/j.cattod.2011.09.007 . hal-01477888

\section{HAL Id: hal-01477888 \\ https://hal.univ-lorraine.fr/hal-01477888}

Submitted on 27 Feb 2017

HAL is a multi-disciplinary open access archive for the deposit and dissemination of scientific research documents, whether they are published or not. The documents may come from teaching and research institutions in France or abroad, or from public or private research centers.
L'archive ouverte pluridisciplinaire HAL, est destinée au dépôt et à la diffusion de documents scientifiques de niveau recherche, publiés ou non, émanant des établissements d'enseignement et de recherche français ou étrangers, des laboratoires publics ou privés.

\section{(1) (1) $\$$}

Distributed under a Creative Commons Attribution - NonCommercial - NoDerivatives $\mid 4.0$ 


\title{
Competitive Diffusion of Gases in a Zeolite Using Proton NMR and a Slice Selection Procedure.
}

\author{
S. Leclerc ${ }^{1}$, M. Petryk ${ }^{2}$, D. Canet ${ }^{1}$, J. Fraissard ${ }^{3, *}$ \\ ${ }^{I}$ Méthodologie RMN, Univ. H. Poincaré, 54506 Vandoeuvre-les-Nancy cedex, France \\ ${ }^{2}$ Modélisation du Transfert de Masse, University Ivan Pul'uy, Ternopil, 46001 Ukraine \\ ${ }^{3}$ LPEM - ESPCI and UPMC, 10 Rue Vauquelin, 75005 Paris cedex, France \\ E-mail: jacques.fraissard@upmc.fr; Tel: 0033609877865
}

Keywords : co-diffusion in zeolite, co-adsorption in zeolite, benzene diffusion in ZSM5, hexane diffusion in ZSM5.

\begin{abstract}
The study of the co-diffusion of several gases through a microporous solid and the resulting instantaneous distribution (out of equilibrium) of the adsorbed phases is particularly important in many fields, such as gas separation, heterogeneous catalysis, etc. Classical ${ }^{1} \mathrm{H}$ NMR imaging is a good technique for the visualization of these processes but, since the signal obtained is not specific for each gas, this requires that each experiment be performed several times under identical conditions, and each time with only one incompletely deuteriated gas. In contrast, we have proposed a new NMR imaging technique (based on the so-called NMR lift) which gives a signal characteristic of each adsorbed gas. It can therefore provide directly, at every moment and at every level of the crystallite bed, the distribution of several gases competing in diffusion and adsorption.
\end{abstract}

\section{Introduction}

Knowledge of the co-diffusion and co-adsorption coefficients of reactants and products is essential when a heterogeneous catalysis reaction is performed by means of a gas flowing through a porous catalyst bed. It can be even more important in a fluidized bed where the contact times between the catalyst pellets and the reactants are very short, which means that one hardly ever knows whether the pellet is used completely or only superficially. Under these experimental conditions the system is never at adsorption equilibrium. The distribution of the various reactants adsorbed on the catalyst is very inhomogeneous and, moreover, very variable from one reactant to another. However, calculations on the kinetics are generally performed by assuming the ideal case of a homogeneous assembly, both for the totality of the catalyst and for the distribution of all the reactants, which makes these calculations, even when they are mathematically rigorous, rather approximate as regards reality. Let us recall, for example, that in the case of a reaction following the Langmuir-Hinshelwood mechanism, the reaction rate is given by the product of the local concentrations of all the reactants, integrated over the entire catalyst. It is therefore necessary to be able to determine at every moment the diffusion coefficient of each reactant in the presence of the others and its instantaneous distribution along the length of the catalyst bed.

Classical ${ }^{1} \mathrm{H}$ NMR imaging is a good technique for the visualization of these processes but, since the signal obtained is not specific for each gas, this requires that each experiment be performed several times under identical conditions, and each time with only one incompletely deuteriated gas. This is very time-consuming and becomes very expensive when completely deuteriated molecules are required. Despite these difficulties it has been previously demonstrated that the distribution of the adsorption of benzene and hexane flowing through a 
ZSM5 bed is very heterogeneous [1]. To remedy the drawbacks of classical imaging we have proposed [2] a new NMR imaging technique which can be used for following the diffusion and adsorption of a gas in a microporous bed [3]. The sample is displaced vertically, step by step, relative to the detector during the adsorption of the gas; the detector is a very thin coil. The bed is assumed to consist of $n$ very thin layers of solid, and the probed region is limited to each layer, so that the variation of the concentration of gas absorbed at the level of each layer is obtained as a function of time. This technique permits the determination of all the parameters of the system at every moment, at every position in the solid and in each crystallite. The complex calculations used to treat the diffusion of benzene alone through a ZSM5 bed have already been published $[3,4]$. An interesting feature of this technique is its ability to visualize directly the co-diffusion of several gases. Indeed, the NMR signal is quantitatively characteristic of the adsorbed gas. It can therefore provide directly, at every moment and at every level of the crystallite bed, the distribution of several gases competing in diffusion and adsorption. We only present here the interest of this technique, without going into the calculation of the resulting diffusion and adsorption parameters.

\section{Experimental}

Our aim is to determine the evolution of the NMR spectrum along the length of a heterogeneous sample in the $\mathrm{z}$ direction of the magnetic field $B_{O}$. This original method of imaging has been presented in [1]. The idea is to select successively several sections at different positions of the sample along the $z$ axis. For this purpose a mechanical device (NMR lift) was built to displace the sample tube (external diameter $5 \mathrm{~mm}$ ) vertically step-bystep inside the NMR probe. In this way one obtains for each position a spectrum corresponding to the composition of the sample at this point. The experiment presented here concerns, as a first example, the co-diffusion of benzene and hexane through a ZSM5 bed (length $15 \mathrm{~mm}$ ). Figure 1 shows the sample-holder tube which is moved vertically, up or down, opposite the very thin detector. The homogeneous liquid solution consists of two equal volumes of benzene and hexane. It is in equilibrium with its gas phase at $25^{\circ} \mathrm{C}$. The two gases begin to diffuse in the zeolite when the glass partition is broken. It is clear that in such an experiment we cannot control all the physical parameters. In order to minimize the temperature variation when the glass wall is broken and the gas comes into contact with the zeolite, the volume under vacuum above the solid is very small and practically negligible compared to the much greater volume of the upper ampoule containing the liquid or gaseous benzene. Moreover, the area of liquid is relatively high, and the diameter of the glass wall to be broken is not small for not disturbing the diffusion of the gas. Finally, because of problems of pollution and of the construction of the NMR probe, we had to use a closed sample-holder tube and not a completely open one. This restriction in no way diminishes the interest of the method.

In accordance with the principle mentioned above, the zeolite sample is displaced vertically in the magnet during the adsorption by $0.5 \mathrm{~mm}$ steps. The signal of the adsorbed gas is detected for each position, rapidly so as to obtain the best possible precision. When the sample reaches the uppermost or the lowest position it starts again in the opposite direction, and continues in this way until adsorption equilibrium is reached. This procedure thus provides the amount of gas adsorption as a function of time for each level in the adsorbent. This makes it possible to determine a diffusion coefficient at each point of the solid [2]. This is essential for tow studies that we are planning : (i) the adsorption of gas in beds consisting of layers of different solids, and (ii) the competitive diffusion of several gases. The latter study will be facilitated by the fact that one detects directly the real characteristic NMR signals. 

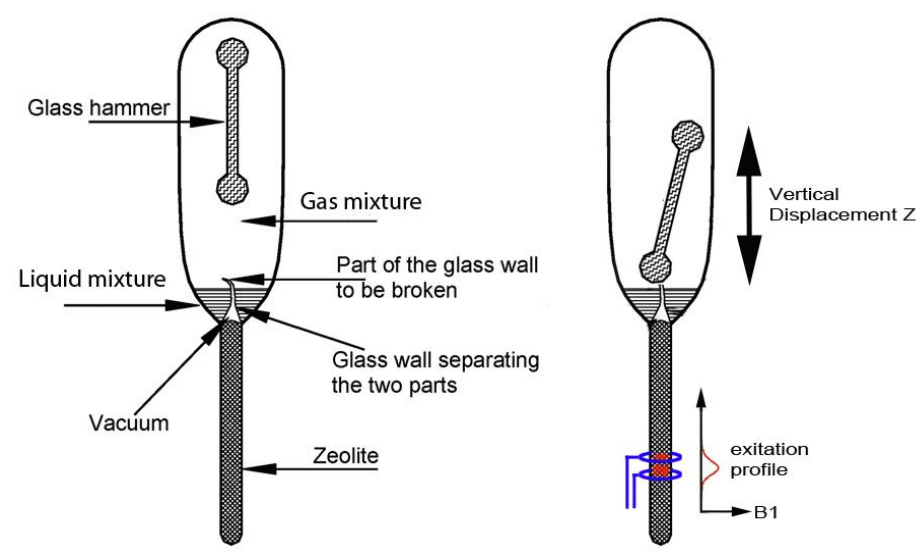

Fig. 1. Left: sample-holder bulb containing the liquid phase in equilibrium with the gas phase;

Right: scheme of the narrow which is zone monitored by the detector.

\section{Results}

Figure 2 shows the ${ }^{1} \mathrm{H}$ NMR spectra of benzene and hexane at two positions, at 6 and 14 $\mathrm{mm}$ (near the bottom of the tube) and after two adsorption times, 34 and 280 minutes. After 34 min. there is still a high concentration gradient the length of the sample. The equalized intensities of the two spectra after $280 \mathrm{~min}$. show that the system has reached equilibrium. To compare the concentrations of adsorbed benzene and hexane molecules, a coefficient, 6/14, has to be applied to the spectrum of the latter to take into account the numbers of $\mathrm{H}$ atoms in each compound. This is displayed in Figures 3 and 4.
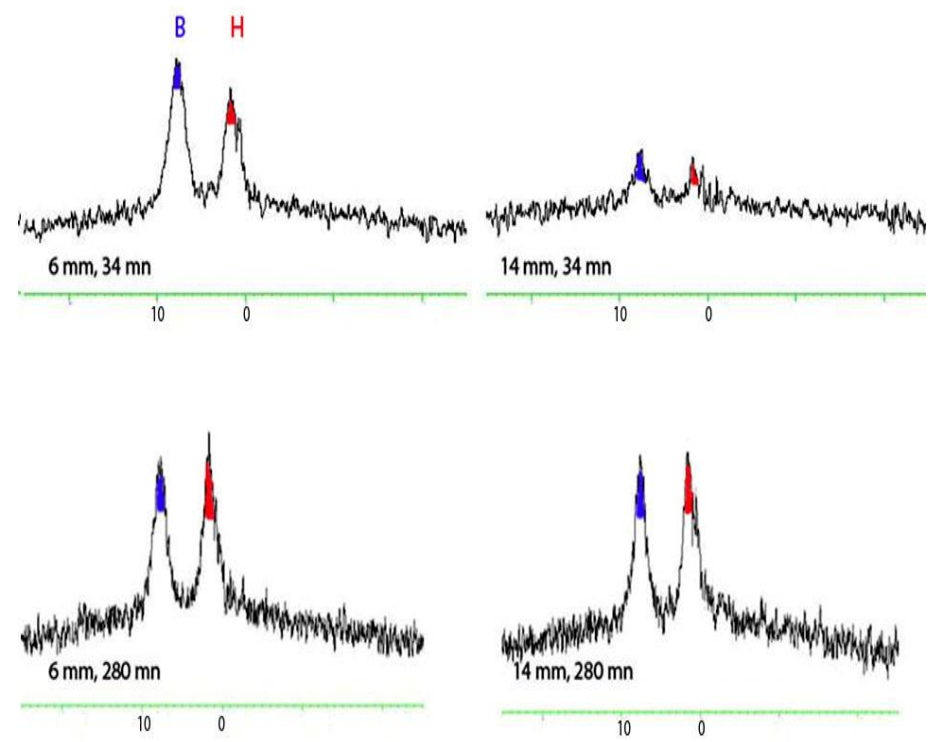

Fig. 2. ${ }^{1} \mathrm{H}$ NMR spectra of benzene (B, 6 atoms) and hexane (H, 14 atoms) at 6 and $14 \mathrm{~mm}$ (from the top of the tube) after 34 and 280 minutes.(The numbers on the abscissa axis are only indicative). 


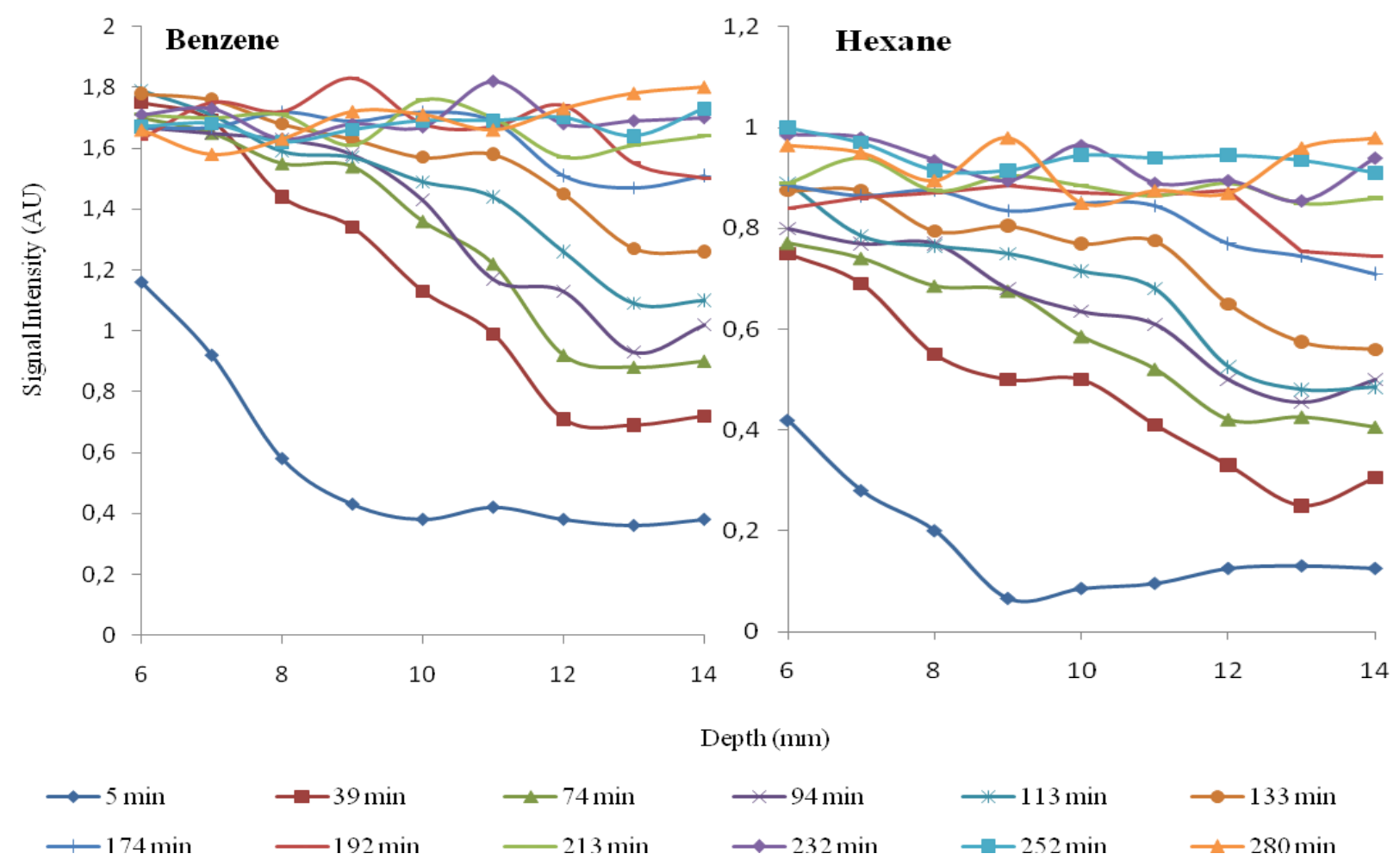

Fig. 3. Benzene and hexane concentrations along the sample, from $6 \mathrm{~mm}$ to $14 \mathrm{~mm}$, for each diffusion time.

Figure 3 presents the benzene and hexane concentrations along the sample, from a height of $6 \mathrm{~mm}$ down to $14 \mathrm{~mm}$, for each diffusion time. We have not considered neither the first diffusion time nor the first layers very close to the gas phase, because it takes 5 to $6 \mathrm{~min}$. from the moment when the glass is broken (therefore the beginning of diffusion) to get the first spectra. It is obvious that after such a time the first layers are saturated, but this restriction does not affect the calculation of the diffusion parameters (not performed here). This figure displays the time dependence of the concentration gradient of each of the gases adsorbed along the catalyst. The signal intensity scale is the same for both components.

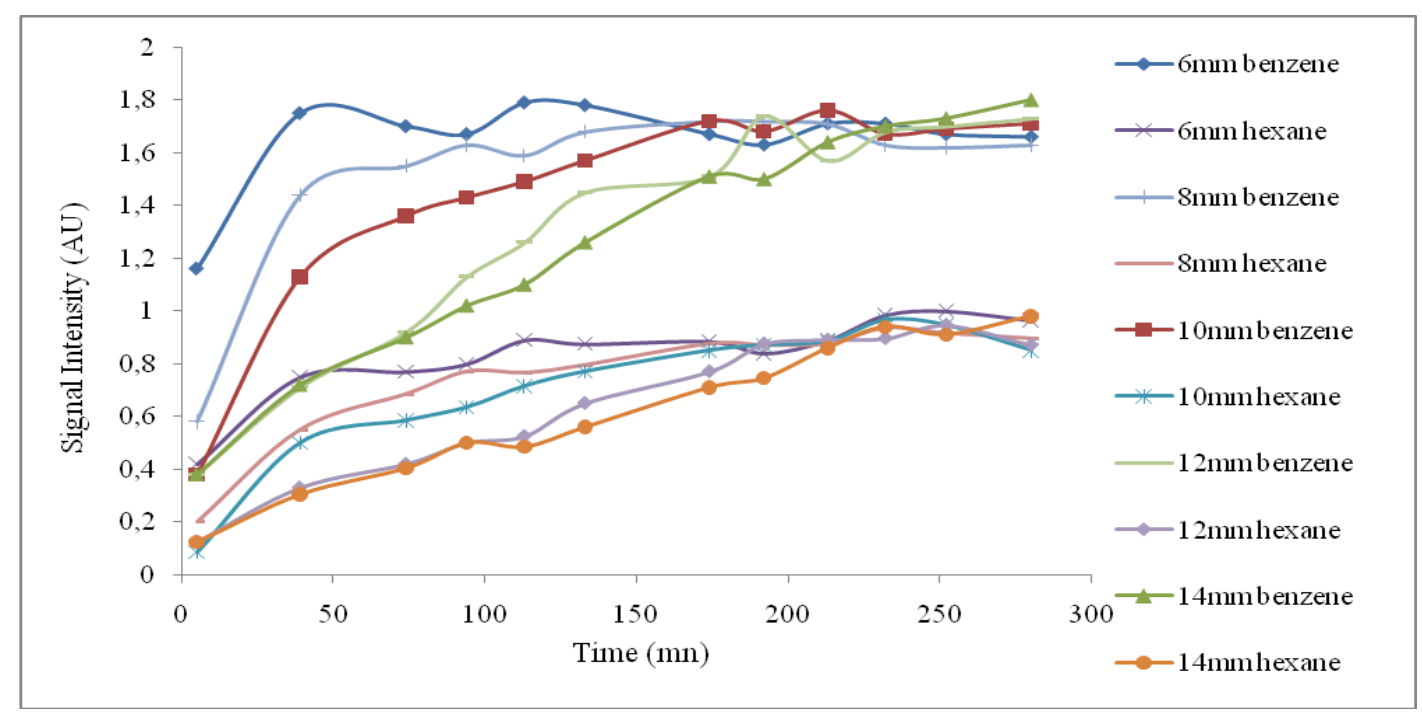

Fig. 4. Evolution, as a function of time, of the benzene and hexane concentrations, at different levels of the sample. 
Figure 4 compares the evolution, as a function of time, of the benzene and hexane concentrations, at different levels of the sample. It reveals particularly well, under the chosen experimental conditions, the hindrance of benzene upon the diffusion of hexane, and this at every moment.

\section{Conclusion}

The choice of the two diffusing gases, benzene and hexane, is not what is most important. In the actual experiment, the system reaches equilibrium and adsorbat homogeneity along the catalyst after a certain time because we had to use a tube closed at the bottom to prevent external pollution. The system will not reach neither equilibrium nor homogeneous distribution when the gases go through a reactor open at both ends, as is generally the case for a reaction. The main result is the possibility, for the first time in a single experiment, of probing at every moment the concomitant distribution of several gases co-diffusing in a porous solid.

\section{References}

[1] P. N'Gokoli-Kekele, M.-A. Springuel, J.-J. Bonardet, J.-M. Dereppe and J. Fraissard Studies in Surface Science and Catalysis, 135, 93 (2001).

[2] S. Leclerc, G. Trausch, B. Cordier, D. Grandclaude, A. Retournard, J. Fraissard, D. Canet, Magn. Reson. Chem. 44 (2006) 311.

[3] M. Petryk, S. Leclerc, D. Canet, J. Fraissard, Catalysis Today 139 (2008) 234.

[4] M. Petryk, S. Leclerc, D. Canet, J. Fraissard, Diffusion Fundamentals 4 (2007) 11.1.

Online in http://www.diffusion-fundamentals.org 
Figure captions

Figure 1. Left: Sample-holder bulb containing the liquid phase in equilibrium with the gas phase; right: Schema of the narrow zone monitored.

Figure 2. ${ }^{1} \mathrm{H}$ NMR spectra of benzene (B, 6 atoms hydrogen) and hexane (H, 14 atoms hydrogen) at 6 and $14 \mathrm{~mm}$ (from the top of the tube) after 34 and 280 minutes.(The numbers on the abscissa do not correspond exactly to the chemical shifts)

Figure 3. Benzene and hexane concentrations along the sample, from $6 \mathrm{~mm}$ to $14 \mathrm{~mm}$, for each diffusion time.

Figure 4. Evolution, as a function of time, of the benzene and hexane concentrations, at different levels of the sample. 